\title{
Médiévales
}

Langues, Textes, Histoire

47 | automne 2004

Îles du Moyen Âge

\section{Les Ysles d'Ynde ou le temps des hommes (Marco Polo - Ibn Battûta)}

\section{Sébastien Douchet}

\section{(2) OpenEdition}

1 Journals

\section{Édition électronique}

URL : https://journals.openedition.org/medievales/505

DOI : 10.4000/medievales.505

ISSN : $1777-5892$

\section{Éditeur}

Presses universitaires de Vincennes

\section{Édition imprimée}

Date de publication : 1 décembre 2004

Pagination : 97-112

ISBN : 2-84292-157-7

ISSN : 0751-2708

\section{Référence électronique}

Sébastien Douchet, «Les Ysles d'Ynde ou le temps des hommes (Marco Polo - Ibn Battûta) », Médiévales [En ligne], 47 | automne 2004, mis en ligne le 02 novembre 2010, consulté le 23 avril 2022. URL : http://journals.openedition.org/medievales/505; DOI : https://doi.org/10.4000/medievales.505

Ce document a été généré automatiquement le 23 avril 2022

Tous droits réservés 


\title{
Les Ysles d'Ynde ou le temps des hommes (Marco Polo - Ibn Battûta)
}

\author{
Sébastien Douchet
}

Ceylan, gemme fuligineuse montée de l'Océan.

(Nicolas Bouvier, Le Poisson-scorpion.)

1 Avec La Description du monde (1298) et la Rihla ${ }^{1}$ (1355) se croisent, à un demi-siècle de distance, les itinéraires des deux plus grands voyageurs du Moyen Âge, Marco Polo et Ibn Battûta. Tous deux ont navigué sur les eaux troubles des ysles d'Ynde ${ }^{2}$ et font le récit de leur voyage ${ }^{3}$ : Marco Polo en observateur tantôt indigné, tantôt amusé, dont l'acculturation asiatique semble avoir rendu sympathique une altérité radicale; Ibn Battûta en observateur désabusé d'un empire musulman en perdition depuis la prise de Bagdad par l'empire Mongol.

2 Sur les franges insulaires de l'Asie, traditionnellement merveilleuses, les deux hommes constatent que ni la loi de la nature ni la loi de Dieu ne sont strictement observées. Les îliens sont les représentants, par leur monstruosité physique et morale, du dévoiement d'une nature humaine que le temps peccamineux de l'Histoire éloigne de son modèle adamique. Mais paradoxalement, et parce que les extrêmes s'y touchent, l'île est aussi lieu des origines : récits, traditions, monuments témoignent d'une Histoire qui remonte au premier pas d'Adam lui-même, puisque c'est à Ceylan que le premier homme aurait posé le pied hors du Paradis... Sur ces terres isolées, coupées du continent ${ }^{4}$, les temporalités se juxtaposent donc, permettant de mesurer l'écart qui sépare l'homme de son archétype et de constater avec étonnement que la nature humaine n'est pas immuable... Mais pourquoi est-ce sur l'île que les récits concentrent de façon privilégiée passé et présent, origine et fantasme, sacré et merveille?

Dans les deux récits, l'insularité n'est pas fondée par la géographie mais par l'imaginaire. Nous formulons donc l'hypothèse que dans ces textes le motif de l'île ne serait pas tant un lieu qu'une figure de l'insularité. Le voyage que firent Marco Polo et Ibn Battûta à Ceylan en est un exemple achevé. En effet, c'est la description d'une montagne, située sur l'île de Ceylan - le Pic d'Adam, autre figure de l'insularité - qui 
illustre de la façon la plus vigoureuse les fonctions dévolues à l'insularité médiévale ${ }^{5}$ : interroger la nature humaine et constater à quel point ses contours sont vacillants.

Ainsi, l'île médiévale créerait les conditions de possibilité d'une réflexion sur la nature humaine. À partir de ce point de vue décalé, il est loisible de constater que la figure de l'île ne décrit pas l'homme, mais dit sa nature. Elle permet l'étiologie plus que la prosopographie ${ }^{6}$. Elle a une fonction herméneutique. Si, comme l'analyse G. Deleuze, l'insularité c'est avant tout le désert qui entoure l'île ${ }^{7}$, il ne s'agit pas du désert maritime que scrutent les Robinsons modernes, mais de ce désert fantasmatique qui arrache le récit au temps ordinaire du monde pour l'inscrire dans le temps suspendu de la réflexion. Comme l'île se dresse à la surface de l'eau, le récit d'île est le miroir où émerge une image de l'Homme.

\section{Une fausse évidence : l'espace insulaire}

5 «Or sachiez que il a en ceste mer ung glouf [golfe] entre l'ysle de Seylam et la terre ferme ${ }^{8}$ La distinction médiévale entre île et continent semble reposer sur une double opposition: l'île n'est pas une terre ferme et le continent n'est pas une terre fermée. Territoire de la clôture, de la circularité et de l'isolement (ce qui constitue un pléonasme étymologique...), l'île est aussi un lieu mal affermi, instable et dangereux. L'opposition semble pertinente pour définir l'identité insulaire. Mais surgit aussitôt un premier paradoxe auquel se heurte tout travail sur l'insularité, qu'il soit géographique, historique, littéraire : dans la mesure où l'île incarne l'instabilité opposée à la terre ferme, ce qui en fonde l'identité est aussi ce qui en rend les contours labiles, changeants et insaisissables. La définition médiévale de l'île elle-même en rend compte, puisque le terme semble pouvoir également désigner les presqu'îles ${ }^{9} .$.

6 Ainsi, à quoi tient-il que ce rivage auquel on aborde soit d'une nature insulaire? Strabon propose une méthode empirique pour qui a déjà débarqué sur l'île : c'est par les sens $\left(\varepsilon \kappa \tau \eta \zeta \alpha l \sigma \ominus \eta \sigma \varepsilon o \varsigma^{10}\right)$ que nous reconnaissons l'insularité d'un territoire; où que nous allions, nous rencontrons la mer. La rencontre perpétuelle de la limite marine garantit d'être sur une île. Mais nos deux récits décrivent les étapes dans les îles et d'îles en îles comme ils feraient des étendues continentales de l'Asie. La référence à la limite maritime est secondaire, voire absente comme dans cet extrait où Marco Polo ne décrit pas mais suggère le cabotage insulaire :

Ore vous avons assez compté de ce royaume, si vous dirons des autres. Et quant l'en se part de ce royaume de Samara, si treuve l'en l'autre royaume qui a a nom Dragroriam $^{11}$.

Comme dans ses étapes continentales, le récit passe de nom en nom plus que d'île en île. Dans la Rihla, Ceylan est dépeinte comme le serait le continent :

Le premier jour, nous campâmes près d'un fleuve que nous avions traversé [...] Puis nous gagnâmes Manâr Mandalî, belle ville située sur la frontière du royaume [...] Nous partîmes pour la petite ville de Bandar Salâwât. Puis nous parcourûmes une région accidentée et très arrosée [...] Nous arrivâmes à la ville de Kunakâr, résidence du principal sultan de la région [...] Nous quittâmes Kunakâr et fîmes halte à une grotte ${ }^{12}$.

7 Le voyage déroule l'espace ceylanais dans une continuité toute continentale. Le cheminement ne conduit pas à un autre rivage qui serait le même que celui qui a été quitté et qui fonderait l'insularité. Étrangement, l'écriture ne rend pas compte d'une expérience de la finitude territoriale de l'île. 
8 Mais si Ceylan est réputée une île, c'est, déclare la Description, parce que «la mappemonde des bons mariniers de celle mer " la signale comme telle et lui attribue " environ .III. mil et .II. ${ }^{C}$ milles ${ }^{13}$ de circonférence. La description de Ceylan et des autres îles n'est pas le fruit d'une expérience "par les sens» du voyageur mais le compte-rendu synthétique - sous la forme d'une mappemonde - d'un savoir coupé de son origine empirique. Lorsque la Rihla indique à propos de l'archipel des Maldives $\mathrm{qu}^{\prime}$ « il y a au moins cent îles, réunies en forme d'anneau $»^{14}$, en aucun cas le récit ne fait état d'un voyage circulaire qui confirmerait ces dires. L'insularité géographique se fonde sur un savoir rapporté, cartographique, et non sur une expérience vécue.

9 L'abordage, moment privilégié de la description de l'île, ne laisse pas de poser problème. Vue depuis le pont du navire, l'île n'est point décrite comme une terre ceinte de mer : le regard du voyageur ne saisit pas l'île dans sa totalité. Voire, il efface du récit la vision de l'île. Ainsi, les basses plaines de Ceylan la rendent invisible pour la vigie :

Maiz saichiez que de la ou vient le vent de tramontane est l'isle toute basse et toute plaine; et quant l'en vient de haulte mer avecques aucune nef l'en ne puet veoir la terre se ce non quant l'en est dessus ${ }^{15}$.

10 Ceylan n'existe que pour celui qui y a débarqué... D'où ce paradoxe : l'île se donne dans l'immédiateté et la présence, tandis que la limite entre terre et mer est effacée, comme si ce critère géographique n'était pas nécessaire à la définition de l'insularité ${ }^{16}$. Le même procédé se retrouve dans la description d'Ibn Battûta qui, depuis le pont du navire, ne voit pas une île mais une montagne dont la vertigineuse verticalité happe le regard vers le haut et le coupe de sa base maritime :

Nous arrivâmes à Ceylan où nous aperçûmes le mont Sarandîb qui s'élevait dans les airs comme une colonne de fumée ${ }^{17}$.

11 La description topographique de l'île ne rend compte ni de sa clôture ni de sa circularité, et l'on ne trouve dans les deux œuvres qu'une exception, fort révélatrice : les Maldives d'Ibn Battûta.

Nous arrivâmes aux îles Maldives qui sont une des merveilles du monde. Il y a au moins cent îles, réunies en forme d'anneau avec une entrée comme une porte par où seulement peuvent entrer les navires ${ }^{18}$.

12 Ces Cyclades de l'Océan Indien pourraient n'être qu'une «merveille » géologique accidentellement disposée à la surface de l'eau. Mais elle est surtout, comme nous allons le constater, un dispositif textuel nécessaire qui révèle la nature profonde de l'île :

Lorsqu'un bateau arrive, il doit se faire conduire par un pilote du pays pour se rendre dans les autres îles. [...] Si le bateau se trompe de direction, il ne peut entrer et le vent l'entraîne vers le Ma'bar ou vers Ceylan ${ }^{19}$.

13 Hors du cercle, les vents perdent les navires au large. Au-dedans, la mer est d'huile, inoffensive. Des pilotes y guident les étrangers et la proximité des îles en fait un univers clos et balisé :

Ces îles sont si rapprochées que la cime des palmiers qui se trouvent dans l'une paraît dès qu'on quitte l'autre ${ }^{20}$.

14 Circonscrire les îles permet de les insérer dans une rêverie sur le repos, l'intimité, la sécurité $^{21}$. Ce contre-exemple est intéressant car il démontre que l'insularité et ses valeurs ne sont pas le fait de l'île. C'est la somme de toutes les îles qui produit un effet d'insularité. L'insularité est donc représentée au second degré, comme c'est toujours le 
cas dans ces récits. L'île n'est pas l'insularité, mais lieu de manifestation de l'insularité. La clôture insulaire n'est pas le fait de son isolement océanique. Elle est un fait narratif.

Ainsi Ibn Battûta, qui fait étape aux Maldives, se voit assigné à résidence par le vizir :

Le vizir m'envoya alors un de ses amis pour me dire: «Le vizir te demande de rester chez nous et tu obtiendras tout ce que tu désires. » Je compris alors que j'étais en leur pouvoir : si je ne restais pas de plein gré, je serais obligé de rester de force et il valait mieux que je le fasse de bonne grâce ${ }^{22}$.

Cette île devient prison non parce qu'elle est entourée de mer, mais parce que le récit en fait le lieu d'une captivité sans échappatoire. Contre toute apparence, elle n'est pas le lieu référentiel et réel du récit de voyage, mais le lieu fonctionnel, propice et nécessaire à la production narrative de caractères discrets qui créent un imaginaire de l'insularité. Ce traitement littéraire de l'archipel des Maldives semble rejoindre les analyses de F. Dubost qui démontre avec justesse que l'île médiévale est le lieu narratif d'une « insularisation » de l'imaginaire ${ }^{23}$.

17 La force et la richesse de cet imaginaire se lisent dans un épisode de la Description où le narrateur évoque l'île de Sypangu :

Et si vous dy que nul n'en oseroit oster n'emporter or de l'isle, pour ce que pou de marchans de terre ferme si vont la pour ce que elle est si loing; si que pour ce ont il tant d'or a desmesure que il n'en scevent que faire. [S'ensuit une description du palais seigneurial dont l'unique matériau est l'or] Ilz ont grant planté de pierres et si ont perles qui sont rouges et sont moult belles et de grant vaillance et qui bien vallent autant comme les blanches et sont moult grosses et rondes et si en ont grant habondance. Et si ont moult grant habondance de pierres precieuses ${ }^{24}$.

L'isolement est source de richesses qui, faute de débouchés marchands, s'accumulent : l'île isole et retient, elle provoque la pléthore, la desmesure et la merveille, c'est-à-dire le prodige qui dépasse l'entendement et provoque l'étonnement. L'île est le lieu du récit où le réel est submergé par la rêverie et le fantasme ${ }^{25}$.

Battûta fait aussi de l'île et de son isolement un support fantasmatique favorisant la rêverie et tirant le sujet hors de sa temporalité propre pour l'emporter vers un irréel du passé, nostalgique et hors d'atteinte :

Nous arrivâmes ensuite dans une petite île de l'archipel où il n'y avait qu'une seule demeure habitée par un tisserand, sa femme et ses enfants et entourée de petits cocotiers. Cet homme possédait une petite barque pour pêcher et se déplacer là où il voulait. Il poussait aussi dans l'île de petits bananiers [...] J'enviais fort cet homme et j'aurais souhaité que cette île m'appartînt pour m'y retirer jusqu'à ma dernière heure ${ }^{26}$.

Embryon utopique plongé dans une temporalité et une expérience qui n'appartiennent pas à l'univers de référence du voyageur, l'île signifie au-delà d'elle-même et ouvre un horizon fantasmatique à l'homme qui aborde à ses rivages.

21 Dans la Rihla et la Description, l'insularité ne se construit donc pas dans la description géographique. Elle ne révèle sa nature qu'indirectement, par des processus narratifs détournés, par une construction souterraine, latente au texte. Elle est avant tout un fait de conscience, le produit d'un imaginaire disséminé, "archipélagique ${ }^{27}$ ", qui se recompose dans le texte par affleurement de figures, d'images, de motifs qui font de l'île le lieu privilégié du récit où peut se dire l'imaginaire insulaire médiéval - un imaginaire polymorphe, aux visages paradoxaux comme peut l'être l'île elle-même, terra non firmata. 


\section{Le Pic d'Adam, paradigme insulaire}

\section{d'îlots sacrés creusés dans la montagne. Le chemin parcouru ne s'évalue alors plus en} distance, mais en sacralité :

Nous fîmes halte à une grotte dite Ustâ Mahmûd al-Lûrî, du nom d'un saint homme qui l'avait creusée au pied de la montagne [...] Nous poursuivîmes avec les Sept Grottes, [...] la grotte d'al-Isfahânî, [...] la grotte de l'oranger amer et celle du Sultan, [...] une grotte qui porte [...] le nom d'Iskandar, [...] la grotte de Khidr ${ }^{32}$. roses dont on prétend qu'elles portent « une inscription portant le nom d'Allah et celui de son Envoyé. ${ }^{33}$ Le chemin est si raide que le pèlerin doit se tenir à dix chaînes successives :

Ces chaînes sont au nombre de dix : deux au pied de la montagne, là où se trouve la porte, sept ininterrompues qu'on voit après les deux premières et la dixième, la chaîne de la shahâda, appelée ainsi parce que le pèlerin qui y arrive et regarde vers le bas est pris d'inquiétude et récite donc la profession de foi pour ne pas tomber ${ }^{34}$.

Le vertige final trouble la conscience du pèlerin comme s'il était frappé de stupeur à l'approche du sacré, tandis qu'espace et texte sacré ne font plus qu'un ${ }^{35}$.

$\mathrm{Au}$ sommet, là où la terre touche le ciel, on visite la trace en creux du pied d'Adam dont les grottes étaient les prémisses insulaires lovés dans la montagne : 
L'empreinte du noble Pied de notre père Adam est restée dans une haute roche noire située dans un vaste espace. Le noble Pied est tant enfoncé dans la roche que l'empreinte est en creux; elle a onze empans de long. Les Chinois sont venus autrefois découper le roc à l'emplacement du gros orteil et la partie qui le touche et l'ont placé dans un temple de Zaytûn [...] L'usage veut que les pèlerins restent trois jours dans la grotte de Khidr et aillent visiter le Pied matin et soir. Et c'est ainsi que nous agîmes ${ }^{36}$.

Le Pic a une triple vocation spirituelle : lieu de contemplation et de commémoration, il est aussi lieu de remémoration des origines et rappelle qu'Adam, homme archétypal, fut premier père, premier saint homme ${ }^{37}$ et premier prophète.

le pied a une double valeur, car il est signe glorieux et fondateur de l'humanité en même temps que signe princeps du péché originel. En effet, le pied en creux, vestige d'une Chute comprise dans son sens le plus concret, matérialise aussi l'entrée de l'homme dans le temps de la finitude. En ce sens, le vol de l'orteil souligne les déprédations et l'outrage que fait subir le passage du temps: le pied mutilé est à l'image de la nature imparfaite de l'homme soumis au temps peccamineux et inexorable de l'Histoire. Voilà sans doute pourquoi la redescente n'emprunte pas le même chemin que celui de l'ascension ${ }^{38}$ et retourne dans l'Histoire par un récit qui dispose dans l'espace les indices d'un temps généalogique :

Nous campâmes dans la grotte de Shaym ou Seth, fils d'Adam. Nous fîmes halte ensuite, près de la baie des Poissons, puis dans les villages de Kurmula, Jabarkâwân, Dildinawa et Âtqalanja. C'est dans cette dernière localité que le cheikh Abû 'Abd Allah ben Khafif [fils de Khafîf] passait l'hiver. Tous les villages et étapes que nous venons de citer se trouvent dans la montagne ${ }^{39}$.

La montagne redevient un lieu habité, humanisé, où l'on peut voir un arbre à propos duquel Ibn Battûta déclare que l'« on raconte des sornettes à son propos comme par exemple celle-ci : le vieillard qui mangerait de ses feuilles retrouverait la jeunesse. C'est tout à fait absurde $! »^{40}$ Le second chemin est bien celui par lequel on revient dans un temps à l'horizon duquel se dessine la mort. La troupe pérégrine arrive alors au niveau de la mer : le voyage peut reprendre son cours horizontal.

Ce pèlerinage constitue donc un moment paradigmatique du récit, qui synthétise les valeurs et fonctions de l'île médiévale. Motif narratif dont la fonction est de servir de cadre à une réflexion sur l'insularité, l'île a une valeur indicielle. Elle fournit au récit un cadre clos et coupé du monde (comme le sommet du Pic) qui permet une sortie du temps de l'Histoire et une entrée dans le temps de la réflexion (ascension) : réflexion sur les origines et le passage du temps (descente). Ainsi, l'insularité de l'île ne dérive pas tant de sa géographie que de sa valeur herméneutique pour penser le temps humain.

\section{Aux fondements de l'insularité : origine, trace et entropie}

Ceylan est lieu de remémoration du passé, de la tradition et de sa transmission : bien avant son pèlerinage, Ibn Battûta dit à plusieurs reprises qu'il ne fait que mettre ses pas dans les pas d'autres fidèles avant d'arriver au Pas ultime, celui d'Adam. Il dessine ainsi l'image d'une communauté qui, depuis cette trace originelle, ne cesse de commémorer le premier père, mais aussi la tradition du pèlerinage elle-même : lors de son passage au mausolée d'Abû 'Abd Allah ben Khafîf en Irak, Ibn Battûta précise que «c'est lui qui 
ouvrit le chemin de la montagne de Sarandîb dans l'île de Ceylan en Inde. ${{ }^{41}}^{41}$ en amont du récit et de l'Histoire se trouve un autre père fondateur qui inventa la route du Pic et dans les pas duquel on marche avant d'arriver au Pas du Père ${ }^{42}$.

\section{Maldives
Mune humar}

L'arbre le plus répandu est le cocotier [...] Le cocotier est surprenant: chacun produit, chaque année, douze régimes qui paraissent à raison d'un par mois. Les uns sont petits, les autres grands, certains sont secs, d'aucuns verts, c'est toujours ainsi [...] Dans une de ces îles, je vis une femme qui n'avait qu'un seul sein; elle avait deux filles : l'une n'avait qu'un seul sein comme sa mère, l'autre en avait deux : l'un très gros et plein de lait et l'autre petit et sec. Je fus fort étonné ! ${ }^{47}$

41 des régimes de noix de coco aussi bien avortons que normaux. Certaines femmes n'échappent pas à la règle et présentent la même anormalité qu'elles transmettent parfois à leurs filles. La monstruosité, prodige de la nature, n'est que la conséquence du délétère passage du temps. L'île suscite donc une interrogation sur la nature humaine dont les contours sont affectés d'un flou étonnant. Ainsi, dans la petite île de Java, l'on 
embaume des singes que l'on fait ensuite passer pour des hommes-pygmées, mystification que dénonce Marco Polo :

Et si vous dy que ceulx qui apportent les petiz hommes qui dient qu'il sont d'Ynde, il dient grant mençonges, car je vous dy que ceulx qui dient qu'il sont hommes d'Ynde si petiz, il ne le sont pas, ains sont synges petiz qui sont contrefaiz en ceste isle en ceste maniere et vous diray comment. Il y a en ceste isle une maniere de synges qui sont moult petiz et ont les visaiges fais comme hommes. Il les prennent et les poillent touz sans ce que il leur lessent ung pou de poil a la barbe et au penyl, et puis les mettent en fourme et les lessent sechier et les adoubent de saffren et d'autres choses si que il semblent a estre hommes ${ }^{48}$.

Mais si le Vénitien rétablit le vrai, il n'en désigne pas moins la similitude troublante entre l'homme et l'animal. Même des hommes se méprennent à la faveur de l'opération taxidermique et ne reconnaissent pas leurs congénères. Toutefois, Marco Polo ne dément que ce qu'il sait et prête foi à des récits merveilleux qui brouillent le statut de la nature humaine :

Et si vous dy que tous les hommes de ceste isle de Angamanam ont chief comme de chiens et dens et yeulx aussi, car il semblent des visaiges tous comme grans chiens mastins $^{49}$.

43 Ce que rapporte également Ibn Battûta :

Nous [...] arrivâmes quinze jours après au pays d'al-Barahnakâr dont les habitants ont des faciès de chiens. Ce sont des sauvages qui ne professent ni la religion des hindous ni aucune autre d'ailleurs ${ }^{50}$.

Les interrogations que suscitent ces récits sont d'autant plus angoissantes que chez Marco Polo se pose le problème de la ressemblance de l'homme à Adam, lui-même créé à l'image de Dieu ${ }^{51} .$. Car de tels peuples aux marges les plus lointaines de l'humanité ressortissent encore à celle-ci. Refoulés aux confins du monde, coupés de la terre ferme, ils jettent le trouble sur la stabilité de la nature humaine.

Il ne faut donc pas s'étonner que dans nos textes la sexualité soit représentée de façon frappante: elle est l'acte premier, originel qui assure reproduction et pérennité du genre humain. Or le fantasme d'une sexualité qui confondrait homme et animal affleure aussi bien dans la Rihla que dans la Description où Marco Polo rapporte à propos des éléphants de Zanzibar :

Sachiez, quand le masle veult habiter a la femelle, si font ung grant fossé en terre et mettent celle femelle dedens a la reverse en celle fosse et l'olifant monte dessus si comme fait l'omme a la fame; et ce est pour ce que elle a sa nature devers le ventre ${ }^{52}$.

L'île enseigne que le privilège du coït face à face, que l'on pensait réservé, n'est pas l'apanage des hommes. Mais ce qui est de l'ordre de l'anecdote chez Marco Polo prend une tournure terrifiante chez Ibn Battûta. Dans les montagnes de Ceylan :

Les singes sont très nombreux dans ces montagnes; ils sont noirs, ont une longue queue et les mâles ont des barbes comme les hommes. Le cheikh 'Uthmân, son fils et d'autres personnes m'ont raconté que les singes ont un chef dont ils sont dépendants, comme si c'était leur souverain. Celui-ci se ceint la tête d'une couronne de feuilles, s'appuie sur un bâton et est entouré, à droite et à gauche, de quatre singes qui tiennent des bâtons. Lorsque le chef s'assoit, les quatre accompagnateurs se tiennent derriere lui. Alors surviennent sa femelle et ses petits qui s'assoient devant lui et cela chaque jour. Les autres singes assistent aussi à cette réunion, mais se tiennent loin du chef. Les quatre accompagnateurs parlent à l'assistance et tous les singes se retirent [...] Un habitant de cette île m'a raconté qu'il avait un de ces singes chez lui. Une de ses filles entra dans une pièce, le singe la suivit. Elle cria 
pour le repousser, mais il la viola. Ils accoururent; le singe accomplissait son œuvre, alors ils le tuèrent ${ }^{53}$. l'homme sur lui-même ${ }^{58}$. En s'écrivant sur son sol, le récit arrache les signes du monde au temps humain et les insère dans une réflexion sur les origines, le temps et la nature humaine ${ }^{59}$. Et bien souvent, l'île fonctionne comme un miroir qui souligne sa puissance réflexive. Miroir topographique (que l'on voie les îles jumelles de Sandur et Condur dont l'une est " grant et l'autre mendre "). ${ }^{60}$ Miroir sociologique aussi, à Sindâbûr :

Trois jours après avoir quitté cette ville, nous fûmes en vue de Sindâbûr, île sur laquelle se trouvent trente-six villages et qui est située dans un estuaire. À la marée descendante, l'eau est douce et bonne à boire, mais à la marée montante elle est salée et saumâtre. Au centre de l'île, sont édifiées deux villes, l'une ancienne construite par les hindous et l'autre par les musulmans, quand ils conquirent l'île pour la première fois ${ }^{61}$.

elève d'un régime double et contradictoire d'un point de vue géographique et humain: elle abrite des peuples aux confessions antagonistes, possède deux pôles urbains, est baignée d'eaux alternativement douces et salées. Ailleurs, les îles Mâle et Femelle séparent les individus d'un même groupe sur deux îles ${ }^{62}$.

dualité ne signifie pas parfaite symétrie : l'île redouble le même de façon affaiblie et amoindrie (hommes vs femmes, grande vs petite île, autochtones vs envahisseurs), ce que le motif du sein fertile et du sein sec illustrait déjà. Cette structure en miroir, où le 
topographique reflète l'humain, indique que l'île est un espace fantasmatique où se révèle une condition humaine dont le sort est de se perpétuer tout en s'éloignant du modèle premier qui est un modèle divin et/ou sacré. De ce point de vue, le double versant du Pic d'Adam oppose le temps de la réflexion abstraite, qui remonte à la contemplation des origines, au temps historique et descendant de l'expérience concrète de la finitude humaine.

Au terme de cette visite dans les ysles d'Ynde, il apparaît donc que la notion d'insularité ne relève pas d'un isolement géographique mais sémiotique. L'île est un signe narratif complexe, un signe au second degré qui, dans le même temps qu'il conte l'ailleurs insulaire, interprète les événements qu'il conte. Voilà pourquoi l'île n'est pas un lieu totalement pris dans le flot continu et banal du temps humain. Émergeant à fleur de monde comme un écueil à la surface de l'eau, l'île, sans doute plus que tout autre lieu, donne à lire de façon privilégiée la part d'ombre et d'imperfection de l'homme. Quant au récit de voyage, il dessine l'image de l'île par résurgences successives, et non par un panorama vu du pont du navire. Une fois encore, l'insularité n'est pas là où on l'attend.

À ce propos, citons une dernière fois Ibn Battûta qui, comme Marco Polo, n'oublie pas de mentionner la richesse de Ceylan en pierres précieuses :

On trouve dans cette ville des corindons superbes, dits al-bahrramân qu'il n'y a que là. Il en est qu'on extrait de la baie et ce sont les plus précieux aux yeux des Cingalais. D'autres sont tirés du sol. À Ceylan, on trouve de ces corindons dans toutes les localités [...]. On achète une concession et on y prospecte. On trouve alors des pierres blanches ramifiées à l'intérieur desquelles s'est formé le corindon. On les remet aux lapidaires qui les polissent jusqu'à ce que la gangue éclate et laisse apparaitre la pierre précieuse. Ces corindons sont rouges, jaunes et bleus, dits naylam. [...] Les concubines du sultan fabriquent avec ces pierres des résilles qu'elles portent sur la tête ${ }^{63}$.

Le sol de l'île gemmifère suffit à résumer l'imaginaire textuel de l'île : des profondeurs du monde le récit d'île extirpe une vérité enfouie qu'il fait briller de mille feux. Ces merveilles alors montées en parure figurent sur la tête des concubines la résille textuelle, archipel de signes qui, d'île en île, dessine sur la mer du temps le profil vacillant d'une humanité énigmatique.

\section{NOTES}

1. Nos éditions de référence sont: Marco Polo, La Description du monde, P.-Y. Badel éd., Paris, 1998 ; Ibn B attûta, Rihla, P. Charles-Dominique éd., Paris, 1995. L'on pourra aussi consulter : Marco Polo, Il Milione, L. Battaglia Ricci éd., Florence, 2001, ainsi que Le Devisement du monde, t. 6, Le Livre de l'Inde, D. Boutet, T. Delcourt, D. James-Raoul éd., Genève, à paraitre en 2006.

2. Description, p. 374. Il s'agit chez Marco Polo des îles qui s'étendent du Japon à Zanzibar en passant par Ceylan: Sypangu (le Japon), Java, les îles Poulo Condor, Bintan, Sumatra, Weh et Nicobar, Andaman, Ceylan, les îles Mâle et Femelle, Socotra, Madagascar, Zanzibar (p. 378-456). Ibn Battûta visite ou évoque quant à lui un ensemble plus restreint, situé autour de la péninsule indienne : Bayram, Sindâbur et un îlot, Hinawr, les Maldives, Ceylan, Andaman, Sumatra, l'« île volante » (p. 902-95). 
3. Pour une première approche de ces textes, outre les éditions citées et leur bibliographie, on pourra consulter M. Guéret-Laferté, Sur les routes de l'empire mongol, Paris, 1994 ; R. Dunn, The Adventures of Ibn Battûta. A muslim traveler of the 14th century, Berkeley, 1986 ; H. Touati, Islam et voyage au Moyen Âge, Paris, 2000.

4. «Insulæ dictæ quod in salo sint, id est in mari » (Isidore de Séville, Etymologice, W. M. Lindsay éd., Oxford, 1989, L. XIV, chap. 6).

5. Sur les affinités entre montagne et îles, on consultera Cl. Thomasset, D. James-Raoul dir., La Montagne dans le texte médiéval. Entre mythe et réalité, Paris, 2000, en particulier J. Ducos, «Entre terre, air et eau: la formation des montagnes" qui note que dans le Speculum naturale la montagne est traitée à la suite des îles (p. 30). Cette classification apparaît déjà chez Isidore de Séville. Cf. n. 9.

6. La prosopographie, en rhétorique, consiste en une description physique d'un personnage.

7. G. Deleuze, «Causes et raisons des îles désertes », dans L'T̂le déserte et autres textes, Paris, 2002, p. 12-17.

8. Description, p. 412.

9. Cf. les Étymologies d'Isidore Séville, L. XIV (De terra et partibus), chap. 6, 7, 8 (« De insulis », « De promuntoriis ", "De montibus»). On lit à propos des presqu'îles: "Commune est insulis ut promineant » (7-1). Pour une analyse plus complète, nous renvoyons à l'article de N. Bouloux dans le présent ouvrage. Relevons que l'iconographie rend également compte de cette plus grande extension sémantique du mot : le ms. BnF fr. 112 (3), qui contient la Queste del saint Graal, comporte une miniature où l'on voit Perceval combattant un dragon dans une presqu'île quand texte et rubrique parlent $\mathrm{d}^{\prime}$ « isle » (fo $\left.23, \mathrm{ca} 1470\right)$.

10. Cité par F. Létoublon, P. Ceccarelli, J. Sgard, «Qu'est-ce qu'une île ?», dans F. Létoublon dir., Impressions d'îles, Toulouse, 1996, p. 10.

11. Description, p. 400.

12. Rihla, p. 943-945.

13. Description, p. 406.

14. Rihla, p. 921.

15. Description, p. 406.

16. L'« île » volante que décrit Ibn Battûta en offre une illustration exemplaire : "Nous vîmes

[...] une montagne en mer. [...] Nous constatâmes, à l'aurore, que cette montagne s'était soulevée au point de laisser passer le jour entre elle et la mer» (p. 994). Si les marins détrompent le voyageur (il ne s'agit pas d'une île, mais de l'oiseau fabuleux Rukhkh), le temps que dure la méprise introduit dans le récit la possibilité prodigieuse qu'existe une île hors des flots. L'on ne saurait mieux formuler à quel point l'insularité de l'île est indépendante de son contexte maritime!

17. Rihla, p. 941.

18. Ibid., p. 921.

19. Ibid., p. 921.

20. Ibid. p. 921.

21. Cf. G. Durand, Les Structures anthropologiques de l'imaginaire, Paris, 1992, p. 287.

22. Ibid., p. 934

23. F. D ubost, "Insularités imaginaires et récit médiéval : l'insularisation », dans J.-Cl. Marimoutou, J.-M. Racault dir., L'Insularité, thématique et représentations, Paris, 1995.

24. Description, p. 378-380.

25. «L'unité de l'île déserte et de son habitant n'est donc pas réelle, mais imaginaire, comme l'idée de voir derrière le rideau quand on n'est pas derrière », G. Deleuze, "Causes... », loc. cit., p. 13.

26. Rihla, p. 941.

27. Ce néologisme est d'Édouard Glissant. Cf. Poétique de la relation, Paris, 1990, p. 46. 
28. Rihla, p. 943.

29. Cf. Coran, Sourate II-36 ; H. GIBB, J. KRAmERS, Shorter Encyclopcedia of Islam, Leyde, 1974, art. "Adam ». Notons que dans l'Islam le pied est le signe de la manifestation divine. Cf. M. Chebel, Dictionnaire des symboles musulmans, Paris, 1995, art. « pied ».

30. Rihla, p. 946.

31. Ibid., p. 946.

32. Ibid., p. 945-947.

33. Ibid., p. 947.

34. Ibid., p. 947

35. Sur les rapports entre île et sacré dans la tradition musulmane, $c f$. A. Zeggaf, « Le Ribât, métaphore de l'île et figure de la spiritualité », dans D. Reig dir., îles des merveilles. Mirage, miroir, mythe, Paris, 1997.

36. Ibid., p. 947-948.

37. Sur le statut de la sainteté dans l'Islam, cf. K. Kreiser dir., Lexikon der islamischen Welt, Stuttgart, 1974, art. « Heiligenverehrung ».

38. Rihla, p. 947.

39. Ibid., p.948. La précision entre crochets provient des Voyages d'Ibn Batoutah, trad. C. Defrémery, B. Sanguinetti, t. 4, Paris, 1872, p. 183. Nous soulignons.

40. Ibid., p. 948.

41. Ibid., p. 564.

42. Dans la tradition musulmane, un autre rocher porte une trace de pied encore plus insigne. Il s'agit du rocher de Jérusalem d'où, d'après la tradition, le Prophète Muhammad lui-même s'élança vers le Ciel. Mais bien qu'il l'ait connue, Ibn Battûta ne fait pas mention de cette trace dans le récit de sa visite au Dôme du Rocher à Jérusalem (cf. Rihla p. 419-20). Cf. O. Grabar, Le Dôme du Rocher. Joyau de Jérusalem, Paris, 1997, p. 43-47.

43. Description, p. 410.

44. G. Deleuze, «Causes... », loc. cit., p. 16.

45. D. Lecoq, "Les Îles aux confins du monde ", dans Îles des merveilles..., op. cit., p. 13-32.

46. Description, p. 406.

47. Rihla, p. 922 et 940.

48. Description, p. 398.

49. Ibid., p. 404.

50. Rihla, p. 964.

51. Cf. Gn I-27 : «Et creavit Deus hominem ad imaginem suam ». En revanche le Coran ne parle pas de ressemblance entre Dieu et sa créature. Cf. Sourate XV-26.

52. Description, p. 454.

53. Rihla, p. 945-946.

54. Cf. la Description où, par exemple, les habitants des îles de Weh et Nicobar « vivent comme bestez », p. 404.

55. Ibid., p. 394.

56. Rihla, p. 949.

57. Cf. Description: sur la petite île de Java «l'estoille de tramontane ne celle du maistre n'apperent de riens ", p. 398.

58. Cf. A. MEISTERHEIM, « Figures... », loc. cit.

59. En cela, l'île est propre à servir de cadre au récit mythique. Cf. F. Moureau dir., L'T̂le, territoire mythique, Paris, 1989, et en particulier A. Abeydeera, "Aspects mythiques de la cartographie de Ceylan de l'Antiquité à la Renaissance », p. 9-18.

60. Description, p. 392.

61. Rihla, p. 902-903.

62. Description, p. 446. 


\section{RÉSUMÉS}

L'île médiévale, telle qu'elle est présentée dans La Description du monde de Marco Polo et la Rihla d'Ibn Battûta, ne se caractérise pas par sa situation géographique, mais par sa valeur herméneutique. Elle offre au récit un cadre coupé du temps du monde où l'imaginaire, sous la forme de la merveille et du monstrueux, questionne l'origine et les fondements de la nature humaine. Cet article analyse par quels procédés narratifs et quelles figures le récit fait des îles de l'Océan Indien un espace privilégié pour explorer les limites vacillantes de l'humanité. En ce sens l'île n'est pas un lieu de l'insularité, mais en est une figure, un signe au second degré.

The Ysles d'Ynde and the Human Time (Marco Polo - Ibn Battûta). The medieval island, as treated in Marco Polo's Description of the world and Ibn Battuta's Rihla, is not characterized by its geographical position, but its hermeneutic value. It gives the narrative a setting, cut off the worldly temporality, where imagination, as merveille and monstrousness, questions the origin and foundations of humankind. This paper analyzes the narrative devices and figures that make the Indian Ocean islands a specific space where the flickering shapes of humankind can be explored. In that way, the island is not a place but a figure, a second-degree sign of insularity.

\section{INDEX}

Mots-clés : Ibn Battûta, insularité, Marco Polo, nature humaine, temps

Keywords : human nature, Ibn Battûta, insularity, Marco Polo, time

\section{AUTEUR}

\section{SÉBASTIEN DOUCHET}

Université Paris X-Nanterre, UFR L. L. Ph., Département de Littérature française, 200, avenue de la République, F-92100 Nanterre 